\title{
Pourquoi l'enseignement de la médecine doit-il évoluer?
}

\section{Pierre-André Michaud}

Prof. Hon., Dr méd., Lausanne

Dans un «billet d'humeur» publié sur la site web de la Faculté de biologie et de médecine, ${ }^{1}$ le Professeur Jean-Daniel Tissot, son Doyen, faisait récemment part de ses doutes quant à la manière de faire évoluer l'enseignement prégradué dispensé aux étudiants en médecine: «Enseignement présentiel, enseignement à distance, objectifs pédagogiques, suivi, évaluations, contrôles: dans tout cela, où est le sens? Où va-t-on?» Il s'interrogeait aussi sur la nécessité d'«enseigner l'économie, l'éthique de la recherche, l'anthropologie, la gestion des données, la globalisation et la mondialisation, la médecine de proximité...».

Ces questions sont importantes, et les doutes exprimés par le Doyen sont probablement partagés par de nombreux enseignants. L'objectif de cet article est d'apporter une réflexion sur la situation actuelle et future de la pratique médicale et la manière dont les facultés de médecine peuvent préparer les étudiants à y faire face, en bref de donner du sens aux changements intervenant dans l'enseignement de la médecine.

\section{Le champ de la médecine en pleine transformation}

Ne disconvenons pas qu'avec l'augmentation du savoir médical, les facultés doivent faire des choix et se centrer sur un objectif majeur qui est de préparer les étudiants à exercer leur métier de médecin assistant muni d'un bon bagage théorique et clinique de base. Néanmoins, les futurs médecins devront aussi savoir s'adapter à un environnement qui change à toute allure. Outre des compétences cliniques absolument essentielles, ils doivent être équipés d'un esprit critique fort et de la capacité de réfléchir aux enjeux techniques, mais aussi sociaux et éthiques que l'évolution du paysage médical suscite.

Au sujet de cet article voir aussi la contribution de Jean Martin à la page 102 de ce numéro.

https://news.unil.ch/ display?id=1524725630064 mesure par exemple, l'irruption du concept de médecine "personnalisée» va-t-elle modifier les pratiques de traitement? Outre les questions éthiques que ces évolutions soulèvent, de telles tendances imposeront aux médecins une capacité d'adaptation hors du commun, ce que les étudiants en médecine semblent d'ailleurs pressentir [1].

Les attentes des patients elles aussi se modifient, avec une augmentation de l'information dont ils disposent notamment grâce à internet (le "health literacy»), et les étudiants doivent apprendre à adopter vis-à-vis de leur patient une démarche favorisant l'autonomie et le partage de la décision [2]. De fait, tout le champ de l'e-health est en train de modifier les rapports que les médecins entretiennent avec leurs patients [3]. Par ailleurs, de plus en plus d'institutions de soins cherchent à répondre aux exigences légitimes des patients en termes de qualité et de sécurité des soins, dont l'une des pierres angulaires est le travail interprofessionnel [4-6]; c'est aussi à une telle approche collaborative que les étudiants en médecine doivent être sensibilisés.

En dehors de toutes ces transformations relatives au champ de la pratique médicale, les futurs médecins doivent enfin être sensibilisés aux modifications de l'environnement démographique et social dans lequel ils évolueront. L'Office fédéral de la santé publique souligne d'ailleurs dans un rapport récent les défis auxquels le système de soins devra répondre [7]: l'escalade des coûts de la santé, l'augmentation des maladies chroniques et de la polymorbidité liée notamment au vieillissement démographique, la pénurie de médecins et de personnel de soin dans certaines disciplines ou régions de notre pays. Ces défis imposent de nouveaux modèles de soins [8], et nécessiteront de la part des médecins des prises de conscience auxquelles les étudiants doivent être préparés. Le développement au sein des facultés de médecine des réflexions portant sur le professionnalisme et l'éthique en est d'ailleurs le témoin $[9,10]$. Comme le relève un récent éditorial de la Revue Médicale Suisse, «l'innovation n'est pas une fin en soi mais un outil de transformation» [11]. 
Innover, transformer, comment répondre à de tels enjeux?

Dans une importante revue publiée il y a quelques années dans le journal Lancet, un groupe international d'experts fait le point sur les enjeux de la formation des personnels de santé [12]. Il relève notamment l'importance de l'émergence du concept d'éducation médicale fondée sur des objectifs d'apprentissage («competency based medical education» [13,14]), une compétence étant définie comme la capacité pour un professionnel d'intégrer dans l'exercice de son métier un ensemble de connaissances, d'attitudes, de valeurs et de pratiques. Ce besoin d'intégration - auquel les étudiants ont de la peine à se faire - a débouché sur l'émergence du concept d'EPA («Entrustable Professional Activity» [15, 16]). L'idée centrale de ce nouveau concept est que les étudiants doivent pouvoir effectuer à la fin de leurs études un certain nombre de prestations cliniques de base de façon autonome; dans cette perspective, les médecins cadres qui les accueillent à l'issue de leurs études devraient pouvoir partir de l'idée qu'ils peuvent leur faire confiance dans un certain nombre de domaines limités. L'apprentissage à l'autonomie se fait évidemment progressivement, dans un premier temps en présence d'un mentor, puis en solo, mais avec un superviseur prêt à intervenir au besoin et enfin sans supervision. D'ailleurs, le concept d'EPA séduit aussi de plus en plus les responsables de formation postgraduée. Le recours aux «entrustable professional activities» est également une manière de sensibiliser les médecins à l'importance de la qualité des soins: disposer d'un solide bagage permettant d'exercer de façon pertinente, scientifique et autonome, en garantissant ainsi la sécurité du patient, constitue un objectif auquel la Loi sur les professions médicales (LPMéd) accorde une importance toute particulière. ${ }^{2}$

En résumé, un curriculum de formation prégraduée équilibré doit faire la part entre l'acquisition de connaissances scientifiques, de compétences cliniques de base solides, bien évaluées, et une sensibilisation accrue au rôle et aux responsabilités du médecin dans notre société, de même que la conquête d'une autonomie et de la juste évaluation de ses propres limites.

2 https://www.admin.ch/ opc/fr/classified-compilation/20040265/201801010 000/811.11.pdf

3 CF: https://www.swissuniversities.ch/fr/themes/enseignement/ reforme-de-bologneects/ $4 \mathrm{http}: / /$ www.profilesmed ch/doc/Profiles_2017.pdf un large degré d'autonomie dans la manière de les conduire. A cela s'ajoute le fait que tous les étudiants, à l'issue de leur master, doivent, pour pouvoir pratiquer dans notre pays, réussir un examen fédéral de médecine comportant deux sessions d'examen par questions à choix multiples ainsi qu'un examen clinique par stations de type ECOS («examen clinique objectif structuré»), dans le cadre duquel les étudiants sont confrontés à des situations cliniques simulées par des acteurs-patients entraînés à cet effet. Enfin, la Loi suisse sur les hautes écoles stipule que toutes les facultés doivent être régulièrement accréditées par des groupes d'experts indépendants, le processus étant piloté par un organisme fédéral, l'AAQ (pour «Swiss Agency of Accreditation and Quality Assurance»). Tous ces aménagements récents résultent de l'adhésion de la Suisse aux accords de Bologne. ${ }^{3}$

Pour assurer une relative homogénéité du profil de compétences des étudiants à la fin de leurs études, la CIMS a décidé de développer non seulement la forme et le contenu de l'examen fédéral, mais également un document qui recense les objectifs d'apprentissage des étudiants en médecine de Suisse, comme cela se fait dans plusieurs autres pays $[17,18]$. Les deux premiers documents, appelés SCLO («Swiss Catalogue of Learning Objectives»), se sont avérés à la longue trop longs et complexes, peu adaptés aux exigences de la LPMéd, à l'évolution de la pratique médicale et aux approches pédagogiques présentées ci-dessus. En conséquence, la CIMS a décidé en 2013 de charger un groupe de travail interfacultés et interprofessionnel de développer un nouveau document. Durant trois ans, soit de 2014 à 2017, une équipe d'experts rassemblant environ vingt cadres académiques issus de toutes les facultés, des représentants de l'Office fédéral de la santé publique et de l'Association suisse des étudiants en médecine (SWIMSA) ont planché sur un manuscrit synthétique d'une quarantaine de pages qui entre en vigueur à l'automne 2018 dans l'ensemble des facultés de médecine de Suisse [19]. Ce document, nommé «PROFILES», pour Principal Objectives For Integrated Learning and Education in Switzerland, est disponible sur internet ${ }^{4}$ et devra guider les responsables pédagogiques, les enseignants et les étudiants dans la manière de préparer au mieux ces derniers à leur formation postgraduée. Il comporte trois chapitres, le premier décrivant les objectifs d'apprentissage que tout étudiant doit avoir atteints, le second proposant neuf activités professionnelles devant être maîtrisées («EPAs») à l’issue des études, et le troisième proposant, dans une même perspective, une liste de situations cliniques courantes, des "Situations as Starting Points». On comprendra que ce fascicule porte essentiellement sur le contenu du master et ne 
recense pas les fondements scientifiques de la médecine qui font la plupart du temps l'objet des enseignements au niveau bachelor.

\section{Et l'avenir?}

On ne saurait conclure ... cela sous-entendrait que le débat est clos. De fait, la mise en place de curricula centrés sur des objectifs et intégrant le nouveau document «PROFILES» est une étape. Les facultés de médecine suisses devront à l'avenir trouver un équilibre entre l'acquisition de connaissances et de compétences de base, et la formation d'étudiants autonomes, capables de s'adapter, sensibles aux aspects économiques, éthiques et sociétaux de l'exercice de la médecine.

- Avec le nombre grandissant d'étudiants et la diminution des durées d'hospitalisation, le travail au lit du malade devient de plus en plus difficile à offrir, mais les facultés doivent créer, avec tous les lieux de soins existants, des réseaux de collaboration qui permettent de conserver cet aspect essentiel de la formation.

- De plus en plus de jeunes assistants se trouvent au début de leur formation postgraduée en situation d'épuisement professionnel. C'est dire l'importance de renforcer au cours des études des occasions pour ces futurs médecins de se préparer à leur rôle, à leurs limites et à l'importance d'un travail d'équipe associant la supervision des aînés, mais aussi la collaboration avec d'autres professions de la santé.

- Le patient doit rester au centre des préoccupations de tous les médecins et soignants: avec la complexification des pathologies et des approches thérapeutiques, avec des exigences accrues en terme d'économicité et d'efficience, les hôpitaux doivent trouver, à travers de nouvelles formes d'organisation du travail, la manière de donner aux cadres le temps de superviser adéquatement les médecins juniors, et la manière pour ces derniers de garder du temps pour la rencontre avec leurs patients.

Tous ces aspects de la formation comportent, selon l'expression consacrée, des menaces et des opportunités. Espérons que les facultés sauront avant tout se centrer sur les opportunités.

Prof. Hon.

Pierre-André Michaud

Sauge 15

CH-1030 Bussigny

pierre-andre.michaud[at]

chuv.ch

\section{Remerciements}

Mes remerciements très sincères vont au Dr Patrick Jucker Kupper, qui a fait une utile révision critique du manuscrit original et en a assuré la traduction en allemand.

\section{Références}

1 Rutberg PC, King B, Gaufberg E, Brett-MacLean P, Dinardo P, Frankel RM. Do Medical Students' Narrative Representations of «The Good Doctor» Change Over Time? Comparing Humanism Essays From a National Contest in 1999 and 2013. Academic medicine: journal of the Association of American Medical Colleges. 2017;92:537-43.

2 Rusiecki J, Schell J, Rothenberger S, Merriam S, McNeil M, Spagnoletti C. An Innovative Shared Decision-Making Curriculum for Internal Medicine Residents: Findings From the University of Pittsburgh Medical Center. Academic medicine: journal of the Association of American Medical Colleges. 2018;93:937-42.

3 Dumiak M. E-health's future frontiers. Bulletin of the World Health Organization. 2012;90:328-9.

4 Crabtree EA, Brennan E, Davis A, Squires JE. Connecting Education to Quality: Engaging Medical Students in the Development of Evidence-Based Clinical Decision Support Tools. Academic medicine: journal of the Association of American Medical Colleges. 2017;92:83-6.

5 Michaud PA, Luthi FT, Clerc M, et al. [Interprofessional education for all ... An awareness experience for undergraduate students in Vaud]. Rev Med Suisse. 2011;7:2363-5.

6 Olson R, Bialocerkowski A. Interprofessional education in allied health: a systematic review. Medical Education. 2014;48:236-46.

7 Federal Office of Public Health. Health 2020: global strategy. Bern 2013.

8 Corrigan P, Parish M. Going with change: Allowing new models of healthcare to be provided for NHS patients. London: Reform; 2014.

9 Doukas DJ, Kirch DG, Brigham TP, et al. Transforming educational accountability in medical ethics and humanities education toward professionalism. Academic medicine: journal of the Association of American Medical Colleges. 2015;90:738-43.

10 Wynia MK, Papadakis MA, Sullivan WM, Hafferty FW. More than a list of values and desired behaviors: a foundational understanding of medical professionalism. Academic medicine: journal of the Association of American Medical Colleges. 2014;89:712-4.

11 Geissbuhler A. Chacun peut faire la différence. Rev Med Suisse. 2018:1531.

12 Frenk J, Chen L, Bhutta ZA, et al. Health professionals for a new century: transforming education to strengthen health systems in an interdependent world. Lancet. 2010;376:1923-58.

13 Powell DE, Carraccio C. Toward Competency-Based Medical Education. The New England journal of medicine. 2018;378:3-5.

14 Ten Cate O, Billett S. Competency-based medical education: origins, perspectives and potentialities. Med Educ. 2014;48:325-32.

15 Ten Cate O. Nuts and Bolts of Entrustable Professional Activities. Journal of graduate medical education. 2013;5:157-8.

16 Chen HC, van den Broek WE, ten Cate O. The case for use of entrustable professional activities in undergraduate medical education. Academic medicine: journal of the Association of American Medical Colleges. 2015;90:431-6.

17 Laan RF, Leunissen RR, van Herwaarden CL. The 2009 framework for undergraduate medical education in the Netherlands. GMS Zeitschrift für medizinische Ausbildung. 2010;27:Doc35.

18 Association of American Medical Colleges. Core Entrustable Professional Activities for Entering Residency. Washington DC: Association of American Medical Colleges; 2014.

19 Michaud PA, Jucker-Kupper P, The Profiles Working G. The «Profiles» document: a modern revision of the objectives of undergraduate medical studies in Switzerland. Swiss Med Wkly. 2016;146:w14270 